\title{
Circadian variation on oxygen consumption in preterm infants
}

\author{
Jacqueline Bauer, ${ }^{1, *}$, Andreas Janecke ${ }^{2}$, \\ Joachim Gerss ${ }^{3}$, Katja Masjosthusmann', \\ Claudius Werner ${ }^{1}$ and Georg Hoffmann ${ }^{4}$ \\ ${ }^{1}$ Department of Pediatrics, University Hospital of \\ Muenster, Muenster, Germany \\ 2 Institute of Medical Biology and Human Genetics, \\ University of Innsbruck, Austria \\ ${ }^{3}$ Institute of Medical Informatics and Biomathematics, \\ University of Muenster, Germany \\ ${ }^{4}$ Department of Pediatrics, University of Heidelberg, \\ Germany
}

\begin{abstract}
Objective: We investigated the diurnal variation in oxygen consumption to determine the optimal time periods of calorimetry in preterm infants.

Methods: Oxygen consumption $\left(\mathrm{VO}_{2}\right)$ was measured continuously for $24 \mathrm{~h}$ using indirect calorimetry. Twentytwo premature infants with gestational age of 27-31 $(31 \pm 1.7)$ weeks were enrolled in the study. Heart rate, respiratory rate, oxygen saturation, skin and rectal temperature and physical activity were monitored continuously.

Results: The averaged values of $\mathrm{VO}_{2}$ showed a significant pattern $(P<0.0001)$ of circadian rhythm with a peak in the afternoon and a nadir during the night with significantly differences between the mean $\mathrm{VO}_{2}$ values. A circadian variation of $\mathrm{VO}_{2}$ was found in more than $80 \%$ of preterm infants and was unrelated to gestational age, physical activity or environmental stress. The infants spent $90.4 \%$ of their time sleeping.

Conclusions: These findings indicate the possible existence of an endogenous circadian rhythm of $\mathrm{VO}_{2}$ in preterm infants beginning shortly after birth. Day-night variations of $\mathrm{VO}_{2}$ should be considered when $\mathrm{VO}_{2}$ values are extrapolated from short measurement periods to prevent overestimation of values.
\end{abstract}

\footnotetext{
${ }^{*}$ Corresponding author: Jacqueline Bauer

Department of Pediatrics

University Hospital of Muenster

Albert-Schweitzer-Straße 34

48149 Muenster

Germany

E-mail: Jacqueline.Bauer@ukmuenster.de
}

Keywords: Circadian variation; oxygen consumption; preterm infants.

\section{Introduction}

Accurate measurements of oxygen consumption $\left(\mathrm{VO}_{2}\right)$ are necessary for calculation of energy metabolism and are helpful to assess energy requirements in preterm infants. It is accepted practice to measure $\mathrm{VO}_{2}$ from several minutes up to a few hours and to extrapolate the values to illustrate a full 24-h period. Several investigations $[17,23]$ examined whether measurement periods of 2-6 $\mathrm{h}$ are sufficient to calculate 24- $\mathrm{h} \mathrm{VO}_{2}$ and energy expenditure (EE). Previous measurements of $\mathrm{VO}_{2}$ have been considered difficult in premature infants, and only few data were published regarding 24-h metabolic measurement to detect day-night variability in the mean $\mathrm{VO}_{2}$ $[19,23]$. Several factors such as frequent feeding and thermal stress make it difficult to obtain standard conditions for measuring $\mathrm{VO}_{2}$ in the extremely immature infant. Three earlier reports [1, 21, 23] showed no diurnal rhythm of $\mathrm{VO}_{2}$ or energy expenditure in neonates, while one group [23] found some circadian variations with maximum EE from 8:00 PM to 2:00 $\mathrm{AM}$ and a minimum EE from 5:00-8:00 AM. A study of 24-h EE measurements in more mature and older infants demonstrated a clear circadian rhythm in EE with low values during the night [19].

Evidence exists that the fetus may become sensitized to a circadian rhythm even before birth. Some reports described an early development of an identifiable pattern of circadian activity [20]. Furthermore, it has been suggested that endogenous perinatal development of circadian rhythms in humans and animals might be based on maturation of the brain more than on exposure to environment [15]. Remarkable examples of circadian patterns such as the sleep-wake cycle and daily rhythms in hormone production have been detected in the last years [22].

In the present investigation, we measured $\mathrm{VO}_{2}$ continuously over $24 \mathrm{~h}$ using indirect calorimetry in preterm infants to explore diurnal variations and to determine the best time periods to obtain $\mathrm{VO}_{2}$ values representative of a 24-h measurement period.

\section{Patients and methods}

Twenty-two preterm infants born at a gestational age of (mean \pm SD) $30.4 \pm 1.7$ weeks, weight $1360 \pm 245 \mathrm{~g}$ at birth, and 
Table 1 Clinical characteristics of the investigated infants.

\begin{tabular}{ll}
\hline Investigated preterm infants $(\mathrm{n})$ & 22 \\
Gestational age (weeks) & $30 \pm 1.7$ \\
Range & $26-32$ \\
Birth weight $(\mathrm{g})$ & $1360 \pm 245$ \\
Range & $970-1690$ \\
Median & 1425 \\
Postnatal age at study time (weeks) & $3.4 \pm 1.7$ \\
Range & $1-6$ \\
Median & 4 \\
Antenatal steroids (\%) & 100 \\
Mechanical ventilation (n) & 3 \\
At study time (\%) & 0 \\
\hline
\end{tabular}

with a postnatal age of $3.4 \pm 1.7$ weeks were enrolled in the study. The characteristics of the study population are shown in Table 1. The study was approved by the Ethics Committee of the University of Heidelberg Medical Center and informed consent was obtained from the parents of all infants. None of the selected infants was intubated, treated with CPAP or supplemental oxygen at the time of study. All infants had no malformations and major current clinical problems. Fifteen infants were treated with caffeine for prevention of apneas and bradycardias using single maintenance doses of $5-10 \mathrm{mg} / \mathrm{kg} /$ day. Serum caffeine concentrations ranged from 10 to $15 \mu \mathrm{g} / \mathrm{mL}$ [4]. To prevent alterations in $\mathrm{VO}_{2}$ with caffeine, all subjects received the medication at the same time. No other stimulating drugs that could alter oxygen consumption were given during the entire study.

All infants were exposed constantly to a regular day-night rhythm in the nursery using a natural, gradual, dawn- and dusk transition of the light-dark cycle from the time of admission to the NICU until hospital discharge. Nursing care and feeding procedures were provided in exactly the same way during the night as during the day to prevent alteration in $\mathrm{VO}_{2}$ caused by the rhythm of activity in the nursery. Throughout the night we used a separate lighting regime for each infant to allow the nurse to individualize lighting according to the needs of the infant.

\section{Monitoring and behavioral state}

Heart rate, respiratory rate, oxygen saturation, skin (lower leg) and rectal temperatures, and physical activity were monitored continuously. All infants were studied in the same type of incubator to achieve thermoneutrality. A modified Freymond Behavioral Scale [9] was used to assess physical activity of the infants throughout the measurement periods. Four different behavioral states were distinguished: I, eyes open or closed, regular respiration, no movements; II, small movements; III, vigorous movements; and IV, crying. All infants were studied, without exceptions, during sleep (state I-II).

\section{Indirect calorimetry}

Measurements of $\mathrm{VO}_{2}$ were performed by means of a portable open-circuit continuous indirect calorimetry device (Deltatrac II Metabolic Monitor; Datex-Ohmeda, Instrumentarium Corp., Helsinki, Finland) as previously reported $[3,5,6]$. The device consists of a differential paramagnetic oxygen sensor and an infrared carbon dioxide sensor. This sensor is attached to a transparent hood which is continuously ventilated by a constant- flow generator, offering the advantage of ready access to the infant. The device measures differences between inspiratory and expiratory oxygen concentrations with accuracy of $0.01 \%$ (vol.). Thus, at a Deltatrac II flow constant of $3 \mathrm{~L} / \mathrm{min}$ (room air and expiration air of the patient), the accuracy in $\mathrm{VO}_{2}$ measurements is $\pm 0.3 \mathrm{~mL} / \mathrm{min}$. Differential measurement is based on repeated automatic zeroing (every $4 \mathrm{~min}$ ) during the measuring sequence. The calibration of the device was performed before each measurement by a standard calibration gas $\left(5 \% \mathrm{CO}_{2}\right.$ and $\left.95 \% \mathrm{O}_{2}\right)$. The analyzer was set zero according to room air. Calibration gases were prepared to an accuracy of $\pm 0.03 \%$ and certified gravimetrically. The Deltatrac II stores each minute-to-minute value of $\mathrm{VO}_{2}$ electronically. At the end of the measurements the values were transmitted to a personal computer and processed using SAS for Windows (SAS Institute, Cary, NC, USA).

\section{Nutrition and study protocol}

Each subject was measured $24 \mathrm{~h}$ continuously to detect diurnal patterns in $\mathrm{VO}_{2}$. Circadian variations of $\mathrm{VO}_{2}$ were defined as a fluctuation of $5 \%$ over baseline values that occur and recur during $24 \mathrm{~h}$ of observation. Measurements began at 8:00 AM in all infants. $\mathrm{VO}_{2}$ was recorded continuously except for the period of nursing and feeding. All 22 premature infants were fed the same preterm formula by gavage every two hours. Each gavage began at full hour (6:00, 8:00 etc.) and lasted for seven to ten minutes. Diaper changes and other nursing procedures lasted for another approximately five minutes. After replacing the canopy a steady state was reached again after $7 \mathrm{~min}$. The interruption time was calculated for 30 min without $\mathrm{VO}_{2}$ measurements. No painful or stressing procedures as needle insertion, heel stick or physiotherapy were performed during the $24-\mathrm{h}$ measurement period.

\section{Statistical analyses}

Each 1-min value of $\mathrm{VO}_{2}$ was recorded, and the 1-min values were used to calculate mean 1-min values for each 10 and 60 -min period. Mean values $\left( \pm S D\right.$ ) of $\mathrm{VO}_{2}$ and activity state were calculated for each 10 and 60-min interval in all 22 infants.

One hour mean values were compared in adjacent intervals by the Wilcoxon signed rank test. Twenty-four hour P-values were Bonferroni adjusted in order to keep the overall error rate alpha at $5 \%$, and were considered significant in the case of $\mathrm{P}<0.05 / 24=0.002$. Power calculations showed that 22 subjects provided a power of at least $75 \%$ of detecting differences in mean values of 0.25 units, assuming a standard deviation of the differences of 0.3 units.

\section{Results}

Figure 1 illustrates the mean 10-min values of $\mathrm{VO}_{2}$ and heart rate for the 22 infants measured throughout the 24-h period. $\mathrm{The} \mathrm{VO}_{2}$ data showed a peak between 2:00 and 5:00 PM, which decreased thereafter from 6:00 PM to 2:00 AM. Another peak was observed between 2:00 $\mathrm{AM}$ and 4:00 AM. Heart rate demonstrated the same trend as $\mathrm{VO}_{2}$ values. The comparison of the $1-\mathrm{h} \mathrm{VO}_{2}$ val- 


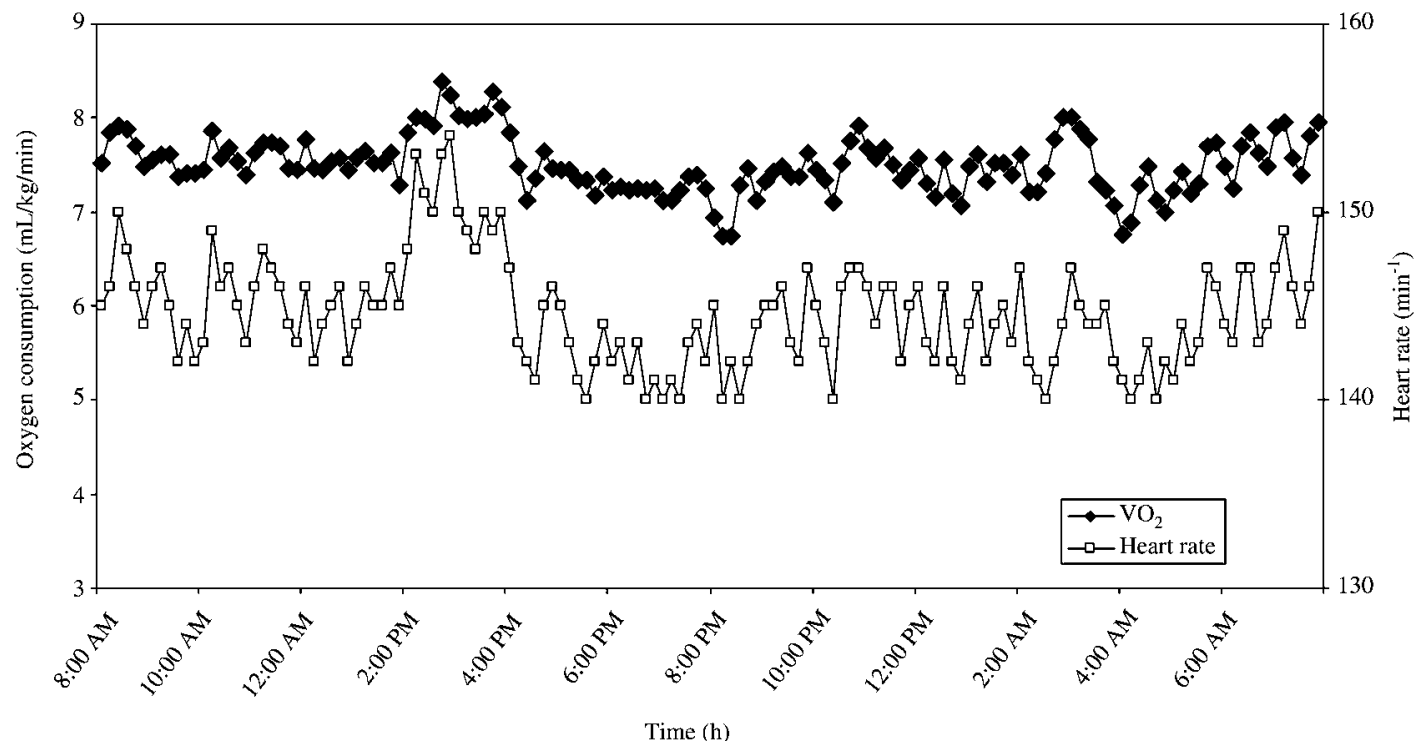

Figure 1 Ten-min values of oxygen consumption and heart rate in a 24-h time period in 22 preterm infants.

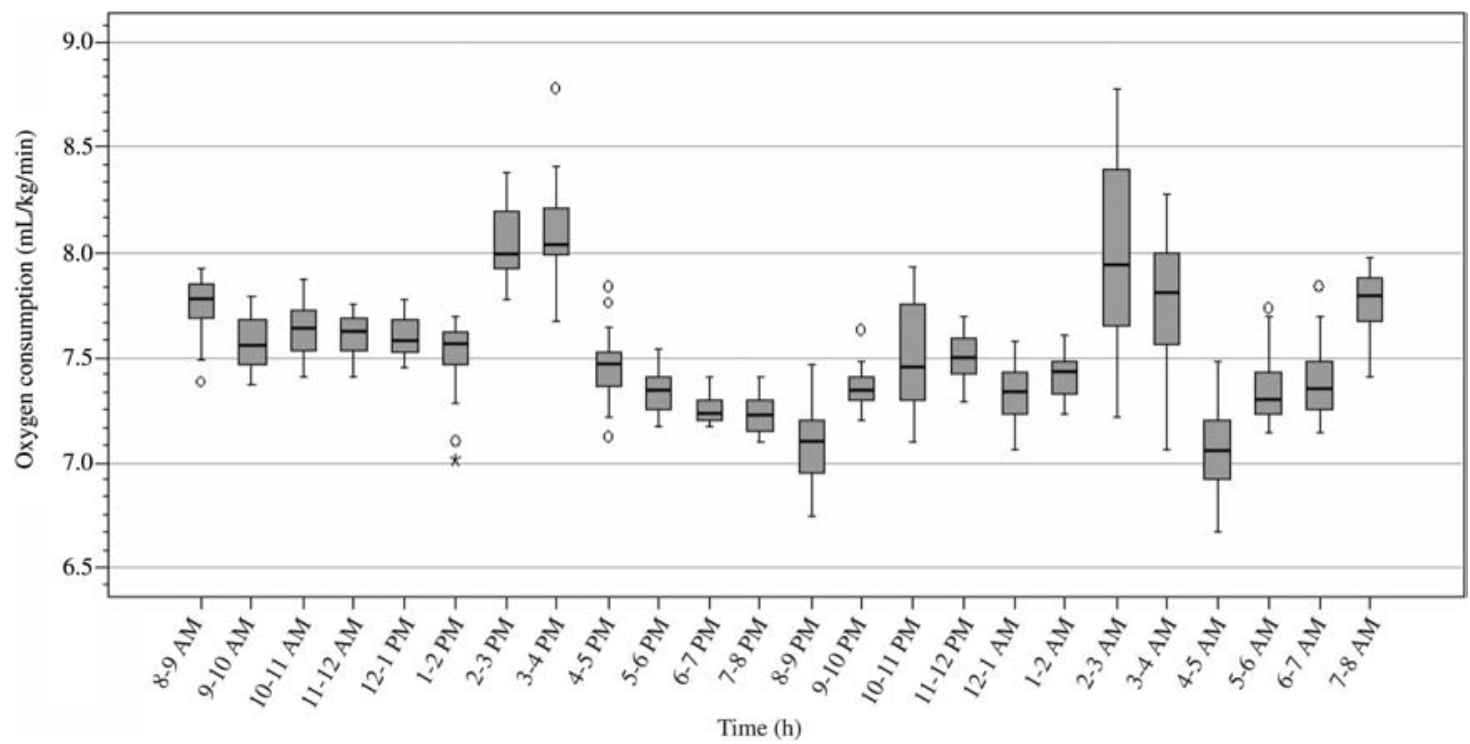

Figure 2 One-hour values of oxygen consumption and heart rate in a 24-h time period in 22 preterm infants.

ues is demonstrated in Figure 2. One-hour values analyses by the Wilcoxon test of all infants confirmed a significant difference $(\mathrm{P}<0.001)$ between the $\mathrm{VO}_{2}$ values in the afternoon and in the early morning. We were able to find evidence of circadian variability in 18 out of 22 preterm infants. In four infants we did not detect any signs of diurnal patterns.

Table 2 demonstrates that the infants were $90.4 \%$ of their time asleep. The $\mathrm{VO}_{2}$ values increased steadily with increasing activity state. There was no significant overall relationship between $\mathrm{VO}_{2}$ and the activity state $(r=0.18)$, whereas $\mathrm{VO}_{2}$ was significantly related to the heart rate $(r=0.58 ; P<0.01)$.
Table 2 Effects of activity states on oxygen consumption in 22 preterm infants. Activity stages according to Freymond et al. [14].

\begin{tabular}{lcc}
\hline Activity state & $\begin{array}{l}\text { Time spent } \\
\text { in this activity }(\%)\end{array}$ & $\begin{array}{l}\text { Oxygen consumption } \\
(\mathrm{mL} / \mathrm{kg} / \mathrm{min})\end{array}$ \\
\hline I & $90.4 \pm 1.1$ & $7.39 \pm 1.3$ \\
II & $6.0 \pm 2.8$ & $8.29 \pm 1.5$ \\
III & $2.5 \pm 1.7$ & $9.12 \pm 2.0$ \\
IV & $1.1 \pm 1.4$ & $10.93 \pm 2.3$ \\
\hline
\end{tabular}

I=eyes closed, regular respiration, no movements; II=eyes closed, irregular respiration, small movements; III=open eyes, no movements; IV = eyes open, gross movements. 


\section{Discussion}

Our study provides evidence for a diurnal variability of $\mathrm{VO}_{2}$ in preterm infants during a 24-h period with a maximum in the afternoon and a smaller peak in the early morning (Figure 1). We detected a diurnal rhythmicity in 18 of the 22 studied premature infants early after birth. The lack of circadian variation in four infants may result from short recordings, and because the infants were measured only once. In addition, many investigators have indicated individual differences in development of circadian rhythms in infants [22].

Schulze et al. [23] studied $\mathrm{VO}_{2}$ in five preterm infants for $24 \mathrm{~h}$ and presented $\mathrm{VO}_{2}$ measurements for eight 3-h intervals. They found the lowest values at 5:00-8:00 AM and higher values from 8:00 PM to 2:00 AM. A clear circadian rhythm in energy expenditure that correlated closely with $\mathrm{VO}_{2}$ was shown in infants with a mean age of five months. The smallest energy expenditure values were during night, from 11:30 PM to 5:30 AM, and the highest values between 6:30 and 11:00 AM [19]. The energy expenditure values were closely related to activity levels.

Short-term variations of $\mathrm{VO}_{2}$ in preterm infants may result from differences in activity, heart rate, breathing pattern and from feeding. Long-term effects on $\mathrm{VO}_{2}$ include postnatal age, weight gain, energy intake, disorders (e.g., chronic lung disease) and the use of some medications $[6,19]$. A strong influence of the activity state on $\mathrm{VO}_{2}$ in preterm infants has been shown by Thureen et al. [26]. In agreement with our results, they observed that preterm infants spent approximately $90 \%$ of the time in active or quiet sleep without major movements. Others $[8,24]$ have found that activity, including different states of sleep, have important influence on metabolic rate. Premature infants are known to sleep $80-90 \%$ of the time, out of which $50-80 \%$ is spent in active sleep [8]. Additionally, it was found that during active sleep $\mathrm{VO}_{2}$ is increasing compared to quiet sleep periods [8, 24]. Observations of rapid eyes movement or EEG were not recorded during our study, but we only detected variations in $\mathrm{VO}_{2}$ in the afternoon and in the early morning independently from sleep stages of the preterm infants. The predominance of low activity state may explain why we did not find significant overall relationship between activity levels and $\mathrm{VO}_{2}$. We demonstrated a significant overall relationship between heart rate and $\mathrm{VO}_{2}$. Glotzbach et al. [10] have shown a circadian rhythm of heart rate resembling the circadian rhythm of $\mathrm{VO}_{2}$. Moreover, some groups $[10,21]$ have reported a marked effect of the heart rate on $\mathrm{VO}_{2}$ during short measurement intervals. The increase in $\mathrm{VO}_{2}$ in preterm infants with chronic lung disease has in part been referred to a rise in heart rate [6].

Feeding has a strong effect on $\mathrm{VO}_{2}$ with maximum values at approximately $15-25$ min after the end of feeding
[25]. We did not measure $\mathrm{VO}_{2}$ during gavage feeding and during the subsequent $20-23 \mathrm{~min}$ after feeding. Thus, we may have missed the rise in $\mathrm{VO}_{2}$ after feeding.

There is some evidence that the fetus may become sensitized to a circadian rhythm even before birth. Circadian rhythms are endogenously generated rhythms that have a period length of about $24 \mathrm{~h}$ and profoundly affect human physiology and behavior [11, 16, 20]. Results from animal models provide information that a biologic clock in the hypothalamic suprachiasmatic nuclei is responsible for the generation of circadian variations [18]. Investigations in preterm and term neonates have described the early development of a recognizable pattern of circadian activity, as well as an early support of a circadian variation in temperature within a week from birth $[12,13]$. Some authors thought that ultradian periodicity was, in the initial stages, much more characteristic than circadian periodicity; others assumed that maternal rhythms of activity had a detectable influence on the fetus in the third trimester [12, 27]. Other investigators reported that circadian rhythms and sleep showed significant development with age, but they failed to find any evidence that exposure to light was the influencing factor $[14,15]$.

Considerable individual variations of measured $\mathrm{VO}_{2}$ values were not observed in our infants, but our data suggest that circadian rhythm in $\mathrm{VO}_{2}$ may begin early after birth with a slight synchronization to daylight cycles. Factors known to influence $\mathrm{VO}_{2}$ such as level of ambient temperature, light and noise were kept constant, but we cannot rule out the possible influence of environmental fluctuations on our results. A limitation of our study is that we did not measure the level of light exposure and we could not control the intensity of noise during the observation period. A correlation between nursing routine or medical procedures and the $\mathrm{VO}_{2}$ peak after midnight could be excluded. Moreover, if a variation in nursing or changes in environmental lighting were responsible for the observed circadian variation, this should have induced a circadian rhythm in the motility of all infants. Both individual variation and the interaction of the individual with environment influences $\mathrm{VO}_{2}$. However, all our studied infants were measured under identical conditions. Previous studies of preterm infants failed to detect diurnal variations of $\mathrm{VO}_{2}$ and the absence of circadian rhythms in the others reports might be due, in part, to very short periods of $\mathrm{VO}_{2}$ measurements, small numbers of infants [7, 23] and different methods and recommendations of perinatal care of the premature infant [2].

We conclude that preterm infants might develop a circadian pattern of $\mathrm{VO}_{2}$ early after birth. These results should be confirmed in a larger group of preterm infants to explore the possibility of diurnal rhythms in very early human development which might indicate a relationship between the presence or absence of circadian rhythms and the level of brain maturation. 


\section{References}

[1] Abdulrazzaq YM, Brooke OG. Respiratory metabolism in preterm infants: the measurement of oxygen consumption during prolonged periods. Pediatr Res. 1984;18:928-31.

[2] American Academy of Pediatrics and American college of Obstetricians and Gynecologists 1997 Guidelines for Perinatal Care, 4th ed. American Academy of Pediatrics and American College of Obstetricians and Gynecologists, Elk Grove Village, IL, and Washington, DC, pp. 47-8.

[3] Bauer J, Fischer Ch, Sontheimer D, Linderkamp O. Metabolic rate and energy balance in very low birth weight infants during kangaroo care by their mothers and fathers. J Pediatr. 1996;129:608-11.

[4] Bauer J, Maier K, Linderkamp O, Hentschel R. Effect of caffeine on oxygen consumption and metabolic rate in very low birth weight infants with idiopathic apnea. Pediatrics. 2001;107:660-3.

[5] Bauer J, Maier K, Hellstern G, Linderkamp O. Longitudinal evaluation of energy expenditure in preterm infants with birth weight below $1000 \mathrm{~g}$. Br J Nutr. 2003;89:533-7.

[6] Bauer J, Maier K, Muehlbauer B, Poeschl J, Linderkamp O. Energy expenditure and plasma catecholamines in preterm infants with mild chronic lung disease. Early Hum Dev. 2003;72:147-57.

[7] Bell EF, Rios GR, Wilmoth PK. Estimation of 24-hour energy expenditure from shorter measurement periods in premature infants. Pediatr Res. 1986;20:646-9.

[8] Darnall RA, Ariagno RL. The effect of sleep state on active thermoregulation in the premature infant. Pediatr Res. 1982;16:512-4.

[9] Freymond D, Schutz Y, Decombaz J, Micheli JL, Jequier E. Energy balance, physical activity, and thermogenic effect of feeding in premature infants. Pediatr Res. 1986; 20:638-45.

[10] Glotzbach SF, Edgar DM, Ariagno RL. Biological Rhythmicity in preterm infants prior to discharge from neonatal intensive care. Pediatrics. 1995;95:231-7.

[11] Korte J, Wulff K, Oppe C, Siegmund R. Ultradian and circadian activity-rest rhythms of preterm neonates compared to full-term neonates using actigraphic monitoring. Chronobiol Int. 2001;18:697-708.

[12] Lohr B, Siegmund R. Ultradian and circadian rhythms of sleep-wake and food-intake behavior during early infancy. Chronobiol Int. 1999;16:129-48.

[13] McGraw K, Hoffmann R, Harker C, Herman JH. The devel- opment of circadian rhythms in a human infant. Sleep. 1999;1;22:303-10.

[14] Mirmiran M, Baldwin RB, Ariagno RL. Circadian and sleep development in preterm infants occurs independently from the influences of environmental lighting. Pediatr Res. 2003;53:933-938.

[15] Mirmiran M, Maas YG, Ariagno RL. Development of fetal and neonatal sleep and circadian rhythms. Sleep Med Rev. 2003;7:321-34.

[16] Panda S, Hogenesch JB, Kay SA. Circadian rhythms from flies to human. Nature. 2002;417:329-35.

[17] Perring J, Henderson M, Cooke RJ. Factors affecting the measurement of energy expenditure during energy balance studies in preterm infants. Pediatr Res. 2000;48:518-23.

[18] Reppert SM. Prenatal development of a hypothalamic biologic clock. Prog Brain Res. 1992;93:119-31.

[19] Rising R, Duro D, Cedillo M, Valois S, Lifshitz F. Daily metabolic rate in healthy infants. J Pediatr. 2003;143:180-5.

[20] Rivkees SA. Developing circadian rhythmicity in infants. Pediatrics. 2003;112:373-81.

[21] Rutter N, Brown SM, Hull D. Variations in the resting oxygen consumption of small babies. Arch Dis Child. 1978;53: 850-4.

[22] Seron-Ferre M, Torres-Farfan C, Forcelledo ML, Valenzuela GJ. The development of circadian rhythms in the fetus and neonates. Semin Perinatol. 2001;25:363-70.

[23] Schulze K, Stefanski M, Masterson J, Kashyap S, Sanocka $U$, Forsyth $M$, et al. An analysis of the variability in estimates of bioenergetic variables in preterm infants. Pediatr Res. 1986;20:422-7.

[24] Stothers JK, Warner RM. Oxygen consumption and neonatal sleep states. J Physiol. 1978;278:435-40.

[25] Stothers JK, Warner RM. Effect of feeding on neonatal oxygen consumption. Arch Dis Child. 1979;54:415-20.

[26] Thureen PJ, Phillips RE, Baron KA, DeMarie MP, Hay WW. Direct measurement of the energy expenditure of physical activity in preterm infants. J Appl Physiol. 1998;85:223-30.

[27] Wulff K, Siegmund R. Emergence of circadian rhythms in infants before and after birth: evidence for variations by parental influence. Z Geburtshilfe Neonatol. 2002;206: 166-71.

The authors stated that there are no conflicts of interest regarding the publication of this article.

Received May 24, 2008. Revised December 12, 2008. Accepted January 26, 2009. Previously published online March 17, 2009. 